\title{
Differential Expression Profiles of the Transcriptome in Breast Cancer Cell Lines Revealed by Next Generation Sequencing
}

\author{
Yu Shi Peng Ye Xinghua Long \\ Department of Laboratory Medicine, Zhongnan Hospital of Wuhan University, Wuhan, China
}

\section{Key Words}

Breast cancer $\bullet$ MCF-7 • MAD-MB-231 • MRNA • MiRNA $・$ LncRNA

\begin{abstract}
Background/Aims: As MCF-7 and MDA-MB-231 cells are the typical cell lines of two clinical breast tumour subtypes, the aim of the present study was to elucidate the transcriptome differences between MCF-7 and MDA-MB-231 breast cancer cell lines. Methods: The mRNA, miRNA (MicroRNA) and IncRNA (Long non-coding RNA) expression profiles were examined using NGS (next generation sequencing) instrument Illumina HiSeq-2500. GO (Gene Ontology) and KEGG (Kyoto Encyclopedia of Genes and Genomes) pathway analyses were performed to identify the biological functions of differentially expressed coding RNAs. Subsequently, we constructed an mRNA-ncRNA (non-coding RNA) targeting regulatory network. Finally, we performed RT-qPCR (real-time quantitative PCR) to confirm the NGS results. Results: There are sharp distinctions of the coding and non-coding RNA profiles between MCF-7 and MDAMB-231 cell lines. Among the mRNAs and ncRNAs with the most differential expression, SLPI, SOD2, miR-7, miR-143 and miR-145 were highly expressed in MCF-7 cells, while CD55, KRT17, miR-21, miR-10b, miR-9, NEAT1 and PICSAR were over-expressed in MDA-MB-231 cells. Differentially expressed mRNAs are primarily involved in biological processes of locomotion, biological adhesion, ECM-receptor interaction pathway and focal adhesion. In the targeting regulatory network of differentially expressed RNAs, mRNAs and miRNAs are primarily associated with tumour metastasis, but the functions of IncRNAs remain uncharacterized. Conclusion: These results provide a basis for future studies of breast cancer metastasis and drug resistance.

\section{Introduction}

Breast cancer is the most common invasive cancer in women, affecting approximately $12 \%$ of females worldwide [1]. In cancer studies, tumour cell lines are widely used as simple models that provide essential tools for more complex biological analyses. MCF-7 and MDA-

Y. Shi and P. Ye contributed equally to this work.

Xinghua Long

KARGER
Department of Laboratory Medicine,

Zhongnan Hospital of Wuhan University, Wuhan, (China)

Tel. +86-27-6781-3517, E-Mail zhoulongxinghua@qq.com 
MB-231 are the most common cell lines used in breast cancer research. These cells both originated from pleural effusions and belong to subtypes of metastatic lobular carcinoma [2]. In principal, the MCF-7 cell line serves as a model of ER+ breast cancers because it expresses oestrogen receptor (ER), making these cells sensitive to oestrogen [3, 4], whereas the MDAMB-231 cell line neither expresses hormone receptors nor human epidermal growth factor receptor-2 (HER-2), thus this cell line is used as a model of triple negative breast cancer (TNBC) [2]. In addition, there are other differences between these two cell lines. MCF-7 cells express markers of luminal epithelial phenotype, while MDA-MB-231 cells show the high expression of vimentin, a known marker of the mesenchymal phenotype. MDA-MB-231 cells are very aggressive while the invasion of MCF-7 cells are relatively weak $[5,6]$.

In the past decade, non-coding RNAs (ncRNAs, in this article only refers to microRNAs and long non-coding RNAs) have become a hot spot in breast cancer initiation and development studies. MicroRNAs (miRNAs) have been implicated in various biological processes, including tumourigenesis, cell proliferation, cell death, stemness and drug resistance [79]. In 2013, an integrated analysis defined miRNA expression signatures that characterize and contribute to the phenotypic diversity of TNBC and its metastasis, and suggested that miRNAs can be effective therapeutic targets for TNBC [10]. Long noncoding RNAs (lncRNAs) have also been demonstrated to influence gene silencing, transcriptional activation, cell cycle, chromosome configuration modification and many other biological functions in breast cancers including TNBC $[11,12]$. Considering these advances in cancer research, additional studies are required to investigate the functions of coding RNAs and ncRNAs in the biological characteristics of cancer.

In the present study, next generation sequencing (NGS) technology was used for the gene profiling analysis of MCF-7 and MDA-MB-231 cell lines. NGS, which is now challenging microarrays as the new choice for transcriptome analysis, offers a more powerful platform to investigate changes of gene expression profiles. Similar topics have previously been analyzed using microarray [13], however, RNA-Seq can overcome the major obstacle of microarray, which is known as cross-hybridization between similar sequences, offer accurate singlenucleotide resolution, which permits the discrimination between highly related sequences, as well as provide increased sensitivity to detect rare sequences [14]. Furthermore, RNASeq has a unique advantage in quantitatively analysing RNA expression levels. Knowing the level of gene expression will help to identify important genes and screen circulating tumour markers. Based on the advantages mentioned above, NGS technology has replaced microarrays as the tool of choice for RNA analysis. In the present study, we uncovered the sophisticated molecular regulation of coding RNAs and ncRNAs in two cell lines, which might be helpful for a better understanding of the biological behaviour of breast cancer cells and provide a platform for further studies for breast cancer metastasis and drug resistance.

\section{Materials and Methods}

\section{Cell lines and reagents}

MDA-MB-231 and MCF-7 cell lines were both obtained from the American Type Culture Collection (ATCC). The cells were grown in RPMI-1640 medium (HyClone) supplemented with 10\% foetal bovine serum (Gibco), $100 \mathrm{U} / \mathrm{mL}$ streptomycin (Gibco), and $100 \mathrm{U} / \mathrm{mL}$ penicillin (Gibco) at $37^{\circ} \mathrm{C}$ in a humidified atmosphere containing $5 \% \mathrm{CO}_{2}$.

\section{RNA preparation and HiSeq2500 sequencing}

The RNA samples were extracted with Trizol. To ensure the good quality of the samples for sequencing, Nanodrop (IMPLEN, CA, USA), Qubit 2.0 (Life Technologies, CA, USA), and the Agilent 2100 Bioanalyzer (Agilent Technologies, CA, USA) were used to detect the purity, concentration and integrity of the RNA samples, respectively. RNA was extracted and identified according to the manufacturer's instructions. MiRNA libraries were generated using the small RNA Sample Library Prep Kit from Illumina (NEB, USA) according to the manufacturer's instructions. LncRNA or mRNA sequencing libraries was generated 


\section{Cellular Physiology Cell Physiol Biochem 2017;44:804-816 \begin{tabular}{l|l|l} 
and BOI: 10.1159/000485344 & $\begin{array}{l}\text { C } 2017 \text { The Author(s). Published by S. Karger AG, Basel } \\
\text { www.karger.com/cpb }\end{array}$
\end{tabular}}

Shi et al.: Differential Expression Profiles of Transcriptome in Breast Cancer Cell Lines

using the NEBNextR UltraTM Directional RNA Library Prep Kit for IlluminaR (NEB, USA) according to the manufacturer's instructions. After construction of the libraries, their concentrations and insert sizes were detected using Qubit 2 and the Agilent 2100 Bioanalyzer, respectively. RT-qPCR was used to accurately quantify the effective concentration and ensure the quality of the libraries. High throughput sequencing was performed on the Illumina HiSeq 2500 sequencing platform.

Quantification of gene expression levels and differential expression analysis

Data filtering was conducted on the raw reads output from the Illumina HiSeq2500 platform, and subsequently clean data were obtained. All downstream analyses were based on high-quality clean data. All clean reads were mapped to the reference human genome GRCh37 (http://grch37.ensembl.org/ Homo_sapiens/Info/Index). The IncRNA symbols were obtained from the Incipedia database. The original sequence counts of miRNAs were normalized to RPM (reads per million), while the original sequence counts of lncRNAs and coding genes were normalized to FPKM (fragments per kilo-base of exon per million fragments mapped). Differential expression analysis of the two samples was performed using the EBseq (2010) R package. Heatmap and hierarchical clustering were performed to generate an overview of the characteristics of expression profiles based on the values of significantly differentially expressed transcripts.

\section{Bioinformatics analysis of $m R N A s$ and $n c R N A s$}

The function of miRNAs is in RNA silencing and the post-transcriptional regulation of gene expression, whereas IncRNAs regulate the expression of neighbouring and overlapping coding genes. Thus, miRNAs and IncRNAs play roles that might be embodied in related mRNA genes. GO analysis (http://www. geneontology.org) was conducted to provide meaningful annotations of genes and gene product properties, and the ontology covers three domains as follows: cellular component, molecular function, and biological process. Additionally, KEGG pathway enrichment analyses were also executed to determine the principal functions of the significantly differentially expressed mRNAs. In addition, we constructed an mRNA-ncRNA targeting regulatory network. First, based on the interactions between IncRNA and its target genes, we used 2 methods to predict the target genes of IncRNA: prediction based on the location relationship between IncRNA and mRNA (the adjacent genes in the range of $100 \mathrm{~kb}$ of lncRNA are the target genes), and prediction based on the complementary base pairing relationship between IncRNA and mRNA (using LncTar target gene prediction tool [15]). Next, the differentially expressed mRNAs targeted by the differentially expressed IncRNAs were collected and a protein-protein interaction analysis was performed. We selected the linked genes for further analysis. Finally, we used TargetScan and Miranda to identiy the miRNAs that regulate these mRNAs, and draw the mRNA-ncRNA target regulating network diagram.

\section{Real-time qPCR validation}

Real-time qPCR (RT-qPCR) was performed to further validate selected differentially expressed coding RNAs and non-coding RNAs from the NGS results. Previously, harvested total RNA from MCF-7 and MDAMB-231 cells was reverse transcribed into cDNA using random hexamer primers (for mRNA and lncRNA) or specific stem-loop RT primers (for miRNA) with a Reverse Transcription Kit (THERMO, USA). Real-time qPCR was performed using the SYBR Green PCR Super Mix (BIO-RAD, USA) and the BIO-RAD CFX96 RealTime PCR Detection System (BIO-RAD, USA). Each sample was analysed in triplicate. The expression levels of mRNA and lncRNAs were normalized to internal control GAPDH, while miRNAs were normalized to U6 snRNA, and subsequently calculated using the $2^{-\Delta \Delta C T}$ method. Statistical analysis was performed using Student's t-test, and $\mathrm{p}<0.05$ was considered statistically significant.

\section{Results}

Overview of differential express characteristics of coding and non-coding RNAs

To explore the differential expression of mRNAs and ncRNAs, gene expression profiles were assessed using NGS. The gene expression of MCF-7 cells was used as a baseline for upor down-regulation of expression in MDA-MB-231 cells. With $\mid \log _{2}$ (Fold-Change) $\mid>=1$ and False Discovery Rate (FDR)<=0.01 as the threshold for significantly differential expression, the results revealed 1036 up-regulated and 871 down-regulated mRNAs, 41 up-regulated 
Table 1. Overview of all differentially expressed non-coding RNAs and coding RNAs (MDA-MB-231 Versus MCF-7)

\begin{tabular}{llll}
\hline RNA species & UP-regulated & Down-regulated & Unchanged \\
\hline mRNA & 1036 & 871 & 10447 \\
miRNA & 41 & 540 & 911 \\
IncRNA & 314 & 247 & 1317 \\
\hline
\end{tabular}

Table 2. The top 15 most abundant transcriptome in MCF-7 and MDA-MB-231 cells respectively

\begin{tabular}{|c|c|}
\hline \multicolumn{2}{|c|}{ MCF-7 cell line } \\
\hline miRNA & $\begin{array}{l}\text { miR-7-5p, miR-423-3p, miR-191-5p, miR-378a-3p, miR-93-5p, miR-22-3p, miR-374b-5p, miR-30e-5p, } \\
\text { miR-125a-5p, miR-320a, miR-16-5p, miR-361-3p, miR-181a-5p, miR-128-3p, miR-143-3p }\end{array}$ \\
\hline IncRNA & $\begin{array}{l}\text { Inc-HLA-C-2, Inc-CCDC57-2, Inc-CSRNP2-2, Inc-NID1-5, Inc-DQX1-1, Inc-WDR4-2, Inc-NCKIPSD-1, } \\
\text { Inc-LPPR5.1-3, Inc-EIF2C2-7, TMEM44-AS1, Inc-COL4A6-2, Inc-SRCIN1-13, Inc-TMEM169-5, Inc-SYF2-3, } \\
\text { Inc-SIRT7-1 }\end{array}$ \\
\hline mRNA & $\begin{array}{l}\text { HIST1H1D, ASS1, SLPI, KRT85, LGALS3BP, HIST1H4L, HIST1H4F, NCCRP1, SPARC, FTH1, SOD2, ARL6IP4, } \\
\text { COL5A2, ISOC2, SRGN }\end{array}$ \\
\hline \multicolumn{2}{|c|}{ MDA-MB-231 cell line } \\
\hline miRNA & $\begin{array}{l}\text { miR-423-3p, miR-21-3p, miR-7-5p, miR-93-5p, miR-378a-3p, miR-10b-5p, miR-191-5p, miR-125a-5p, } \\
\text { miR-30e-5p, miR-374b-5p, miR-16-5p, miR-361-3p, miR-181a-5p, miR-423-5p, miR-320a }\end{array}$ \\
\hline IncRNA & $\begin{array}{l}\text { Inc-KCNJ8-2, Inc-AC007952.2.1-2, Inc-VAMP2-5, Inc-CCNB1IP1-4, PICSAR, Inc-ARMCX6-2, Inc-ATP5B-5, } \\
\text { Inc-DHX57-3, Inc-RHOG-3, Inc-PIEZO2-14, NEAT1, Inc-C12orf77-1, Inc-NFRKB-4, Inc-B3GNT8-1, Inc-USP45-2 }\end{array}$ \\
\hline mRNA & RPS29, FTH1, RRM1, IGFBP7, LUM, ITGB1, DCN, DUSP1, CD55, SPINK6, KRT17, JUNB, CYR61, CTGF, BZW1 \\
\hline
\end{tabular}

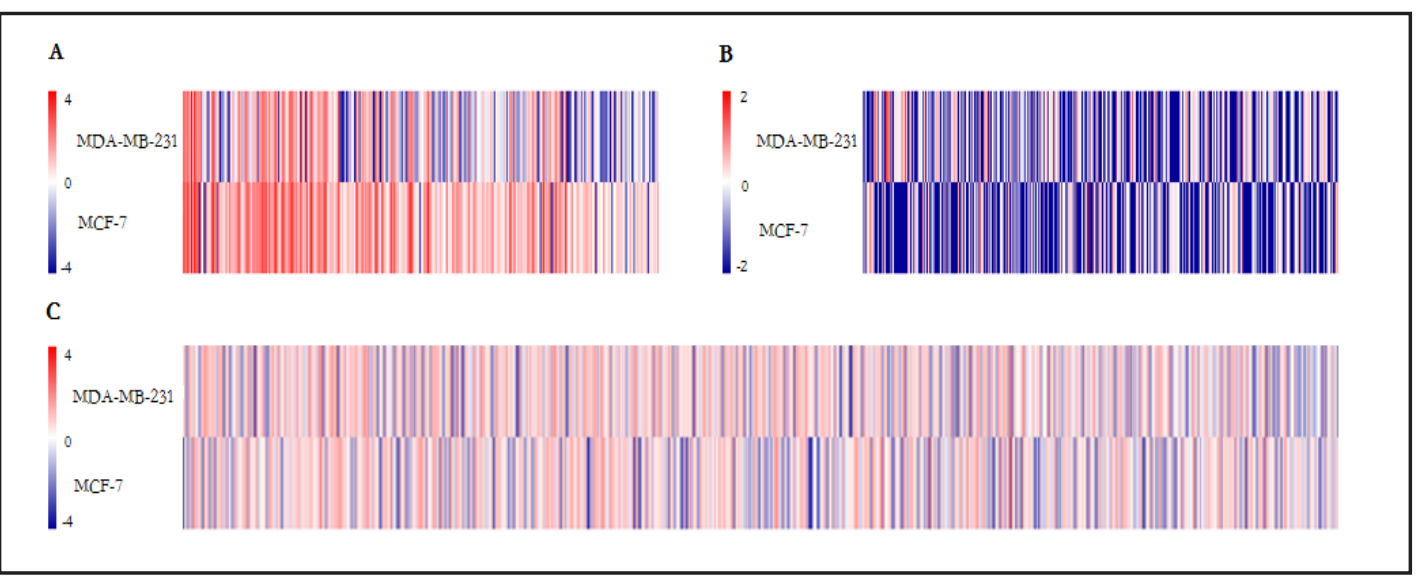

Fig. 1. Heatmap of differentially expressed miRNAs (A), IncRNAs (B) and mRNAs(C) in MDA-MB-231 comparing with MCF-7 cell line. The colour bar in (A) refers to the $\log _{10}$ FPKM of miRNA, the colour bar in (B) and (C) refers to the $\log _{10}$ RPM of lncRNA and mRNA. "Red" indicates high relative expression, and "blue" indicates low relative expression.

and 540 down-regulated miRNAs and 314 up-regulated and 247 down-regulated lncRNAs (Table 1). The heatmap (Fig. 1) provides a visual representation of the differences in the RNA expression levels between MDA-MB-231 and MCF-7 cells. RNA expression levels have been widely studied by many researchers. In Table 2, we listed the top 15 mRNAs and ncRNAs expressing the highest level in two cell lines, respectively. Furthermore, we list the most differentially-expressed transcriptomes in Tables 3 and 4. MiR-143, miR-30e, miR-7, etc. were highly expressed in MCF-7 cells, whereas miR-21 and miR-10b were highly expressed in MDA-MB-231 cells. According to the result of IncRNA differential expression, lnc-HLA-C-2,

\section{KARGER}




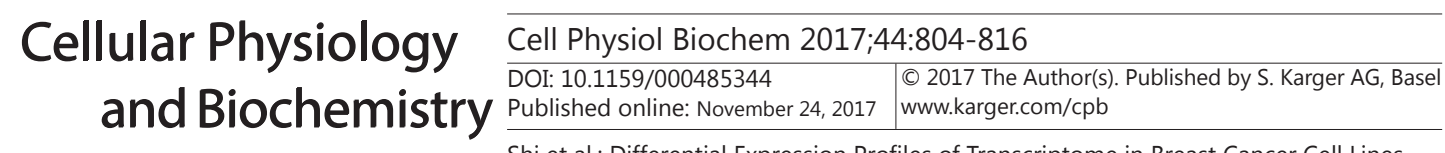
808

Shi et al.: Differential Expression Profiles of Transcriptome in Breast Cancer Cell Lines

Table 3. The top 15 most up- and downregulated miRNAs revealed by NGS. The table lists the top 15 up- and downregulated miRNAs based on the fold change values. These miRNAs are dominantly expressed. $\log _{2}$ FC: $\log _{2}$ Fold Change.

\begin{tabular}{|c|c|c|c|c|c|c|c|c|c|}
\hline miRNA & $\begin{array}{l}\text { MCF- } \\
7\end{array}$ & $\begin{array}{l}\text { MDA-MB-2 } \\
31\end{array}$ & $\begin{array}{l}\log _{2} F \\
C\end{array}$ & $\begin{array}{l}\text { Up/Dow } \\
n\end{array}$ & miRNA & MCF-7 & $\begin{array}{l}\text { MDA-MB-2 } \\
31\end{array}$ & $\begin{array}{l}\log _{2} F \\
C\end{array}$ & $\begin{array}{l}\text { Up/Dow } \\
n\end{array}$ \\
\hline $\begin{array}{l}\text { miR-10b-5 } \\
p\end{array}$ & 4.7 & 1378.5 & 8.2 & Up & $\begin{array}{l}\text { miR-548ah- } \\
3 p\end{array}$ & 180.7 & 1.9 & -6.6 & Down \\
\hline $\begin{array}{l}\text { miR-296-3 } \\
p\end{array}$ & 2.6 & 58.5 & 4.5 & Up & miR-4521 & 1019.4 & 11.3 & -6.5 & Down \\
\hline $\begin{array}{l}\text { miR-675-3 } \\
p\end{array}$ & 7.1 & 116.7 & 4.1 & Up & miR-548p & 173.3 & 2.5 & -6.1 & Down \\
\hline miR-1-3p & 12.4 & 160.2 & 3.7 & Up & miR-143-3p & 1647.8 & 26.6 & -6.0 & Down \\
\hline miR-9-5p & 12.7 & 149.8 & 3.6 & Up & miR-424-3p & 549.0 & 14.4 & -5.2 & Down \\
\hline $\begin{array}{l}\text { miR-296-5 } \\
p\end{array}$ & 1.5 & 11.6 & 3.0 & Up & miR-145-5p & 128.7 & 3.9 & -5.0 & Down \\
\hline miR-4473 & 3.8 & 23.4 & 2.6 & Up & $\begin{array}{l}\text { miR-1249-3 } \\
p\end{array}$ & 48.1 & 1.6 & -4.9 & Down \\
\hline$m i R-21-3 p$ & $\begin{array}{l}495 . \\
8\end{array}$ & 2536.4 & 2.4 & Up & $\begin{array}{l}\text { miR-200c-3 } \\
p\end{array}$ & 332.5 & 12.1 & -4.8 & Down \\
\hline $\begin{array}{l}\text { miR-210-3 } \\
p\end{array}$ & 48.1 & 205.0 & 2.1 & Up & $\begin{array}{l}\text { miR-548h-5 } \\
p\end{array}$ & 64.1 & 2.5 & -4.7 & Down \\
\hline $\begin{array}{l}\text { miR-335-3 } \\
p\end{array}$ & 6.8 & 20.0 & 1.6 & Up & miR-573 & 35.1 & 1.4 & -4.7 & Down \\
\hline $\begin{array}{l}\text { miR-675-5 } \\
p\end{array}$ & 18.9 & 55.0 & 1.5 & Up & miR-1303 & 602.4 & 24.8 & -4.6 & Down \\
\hline miR-217 & 5.0 & 14.2 & 1.5 & Up & miR-7-5p & $\begin{array}{l}57810 . \\
1\end{array}$ & 2444.6 & -4.6 & Down \\
\hline $\begin{array}{l}\text { miR-196-5 } \\
p\end{array}$ & $\begin{array}{l}106 . \\
0\end{array}$ & 253.3 & 1.2 & Up & miR-22-3p & 5449.1 & 267.5 & -4.3 & Down \\
\hline miR-7706 & 79.7 & 183.7 & 1.2 & Up & miR-129-5p & 78.2 & 4.7 & -4.1 & Down \\
\hline $\begin{array}{l}\text { let-7f-2-3 } \\
p\end{array}$ & 6.8 & 15.5 & 1.2 & Up & $\begin{array}{l}\text { miR-181a-3 } \\
p\end{array}$ & 538.0 & 32.5 & -4.0 & Down \\
\hline
\end{tabular}

Table 4. Most differentially-expressed mRNAs and IncRNAs revealed by NGS. The table only lists the top 6 up- and downregulated mRNAs and lncRNAs based on the fold change values. These mRNAs and lncRNAs are dominantly expressed. $\log _{2}$ FC: $\log _{2}$ Fold Change

\begin{tabular}{llcccc}
\hline mRNA/IncRNA & Gene symbol & MCF-7 & MDA-MB-231 & log 2 FC $_{\text {Up/Down }}$ \\
\hline mRNA & CTGF & 0.3 & 213.9 & 9.2 & Up \\
mRNA & SLC43A3 & 0.1 & 37.1 & 8.0 & Up \\
mRNA & ACKR3 & 0.1 & 34.8 & 7.9 & Up \\
mRNA & EREG & 0.2 & 55.2 & 7.5 & Up \\
mRNA & MBNL3 & 0 & 7.6 & 7.5 & Up \\
mRNA & CDYL2 & 0.1 & 24.7 & 7.4 & Up \\
mRNA & SPARC & 184.0 & 0.2 & -9.6 & Down \\
mRNA & HIST1H4F & 227.7 & 0.2 & -9.0 & Down \\
mRNA & COL5A2 & 138.0 & 0.3 & -8.6 & Down \\
mRNA & VAV3 & 28.9 & 0.1 & -8.0 & Down \\
mRNA & TUSC3 & 46.3 & 0.1 & -7.9 & Down \\
mRNA & IGFBP2 & 83.0 & 0.2 & -7.8 & Down \\
IncRNA & PICSAR & 0.1 & 18.5 & 6.1 & Up \\
IncRNA & Inc-PITPNM2-1 & 0 & 1.3 & 4.5 & Up \\
IncRNA & Inc-RHOG-3 & 0.2 & 9.3 & 4.3 & Up \\
IncRNA & Inc-PPIL1-2 & 0.2 & 4.0 & 4.0 & Up \\
IncRNA & GAS5 & 0.2 & 5.4 & 3.9 & Up \\
IncRNA & Inc-CCNB1IP1-4 & 1.6 & 19.3 & 3.5 & Up \\
IncRNA & Inc-NCKIPSD-1 & 8.9 & 0.1 & -5.4 & Down \\
IncRNA & Inc-RP11-247C2.2.1-6 & 3.2 & 0.1 & -4.7 & Down \\
IncRNA & LINC00473 & 1.4 & 0 & -4.5 & Down \\
IncRNA & Inc-NID1-5 & 11.9 & 0.5 & -4.1 & Down \\
IncRNA & CTD-2184D3.5 & 4.1 & 0.2 & -3.8 & Down \\
IncRNA & Inc-GOLM1-2 & 1.6 & 0.1 & -3.8 & Down \\
\hline & & & & & \\
\hline
\end{tabular}




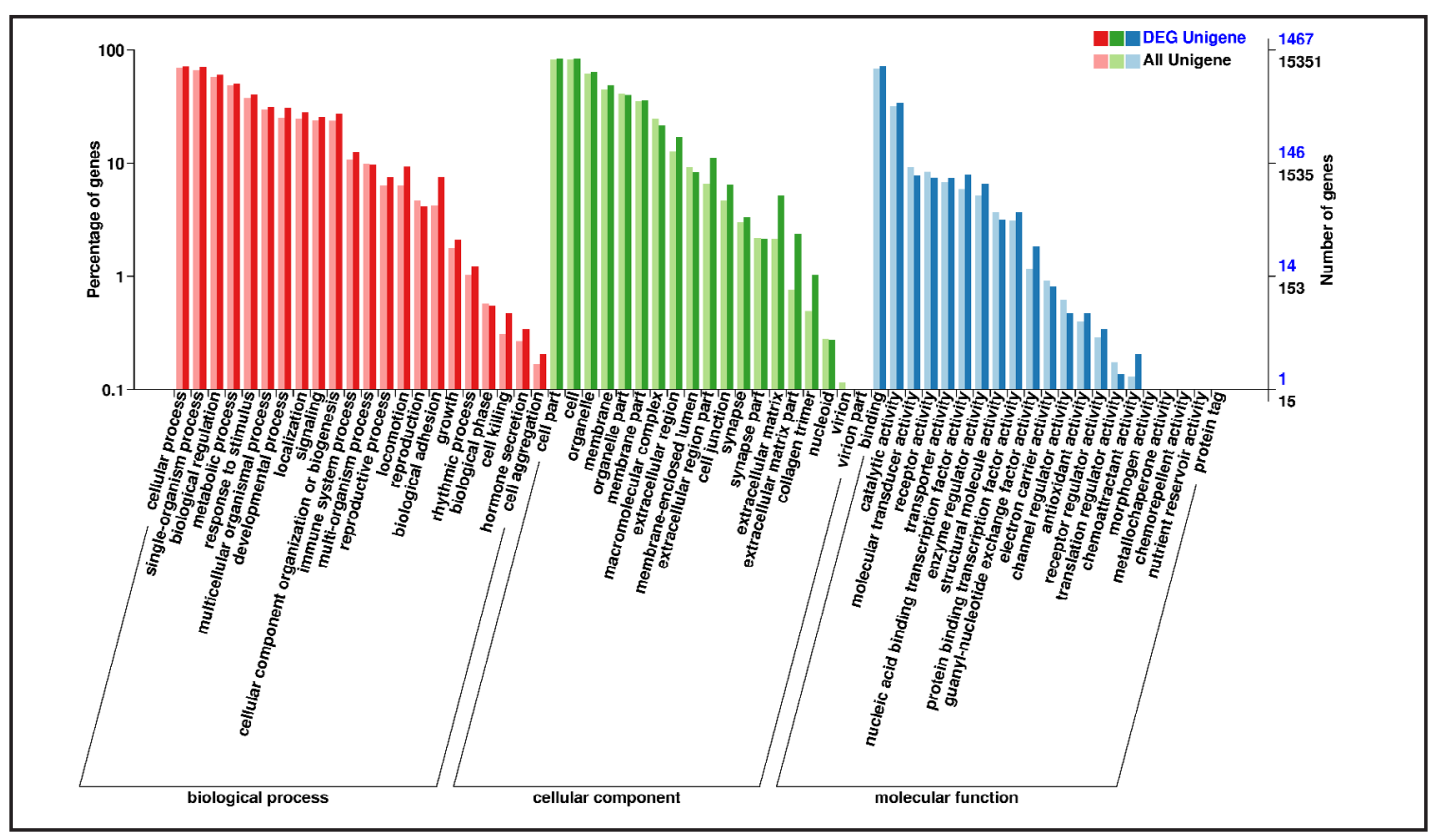

Fig. 2. Gene ontology analysis of differentially expressed mRNAs. GO classifications are shown on the horizontal axis, percentages of gene counts are shown on the left vertical axis and gene counts are shown on the right. This figure shows the gene enrichment of the GO sub-level function in the backgrounds of differentially expressed genes and all genes. The results reflects the status of different sub-level functions in two backgrounds. The sub-level function which shows significantly different proportions indicates different enrichment between differentially expressed genes and all genes.

lnc-CCDC57-2, lnc-CSRNP2-2, etc. were highly expressed in the MCF-7 cell line. Additionally, PICSAR and NEAT1 showed higher expression in the MDA-MB-231 cell line than in the MCF7 cell line. The most abundant mRNAs in MCF-7 included SLPI, SOD2, etc., whereas the most abundant mRNAs in MDA-MB-231 were CD55, KRT17, etc.

\section{GO and KEGG pathway analysis of differentially expressed coding RNAs}

To better understand the functions of the differentially expressed mRNAs described above, we performed GO and KEGG pathway enrichment analyses. Approximately 1467 differentially expressed mRNAs obtained Gene Ontology annotations. The GO analysis of these mRNA expression profiles showed that these genes were involved in the biological process of locomotion, biological adhesion and cell killing; primarily enriched in extracellular matrix, extracellular matrix part and collagen trimer about cellular components; and involved in molecular functions, including guanyl-nucleotide exchange factor, chemoattractant and enzyme regulator activities (Fig. 2). Additionally, 1184 mRNAs obtained KEGG annotation. The top 20 pathways with the most significant enrichment are listed in Fig. 3. Remarkably, these mRNAs were enriched in ECM-receptor interaction pathway, pathway in cancer, focal adhesion, MAPK signalling pathway, TNF signalling pathway and PI3K-Akt signalling pathway, etc.

\section{Targeting regulatory relationships of $m R N A$ s and ncRNAs}

We predicted the target mRNAs of the differentially expressed lncRNAs and examined the intersection of these mRNAs and the differentially expressed mRNAs in the present study. We finally obtained a total of 133 mRNAs. The String database was used to analyse the protein-protein interactions of the proteins encoded by these mRNAs, and 9 differentially expressed RNAs were selected as the hub genes of this network. Finally, we constructed a mRNA-ncRNA targeting regulatory network according to the targeting regulatory relation- 


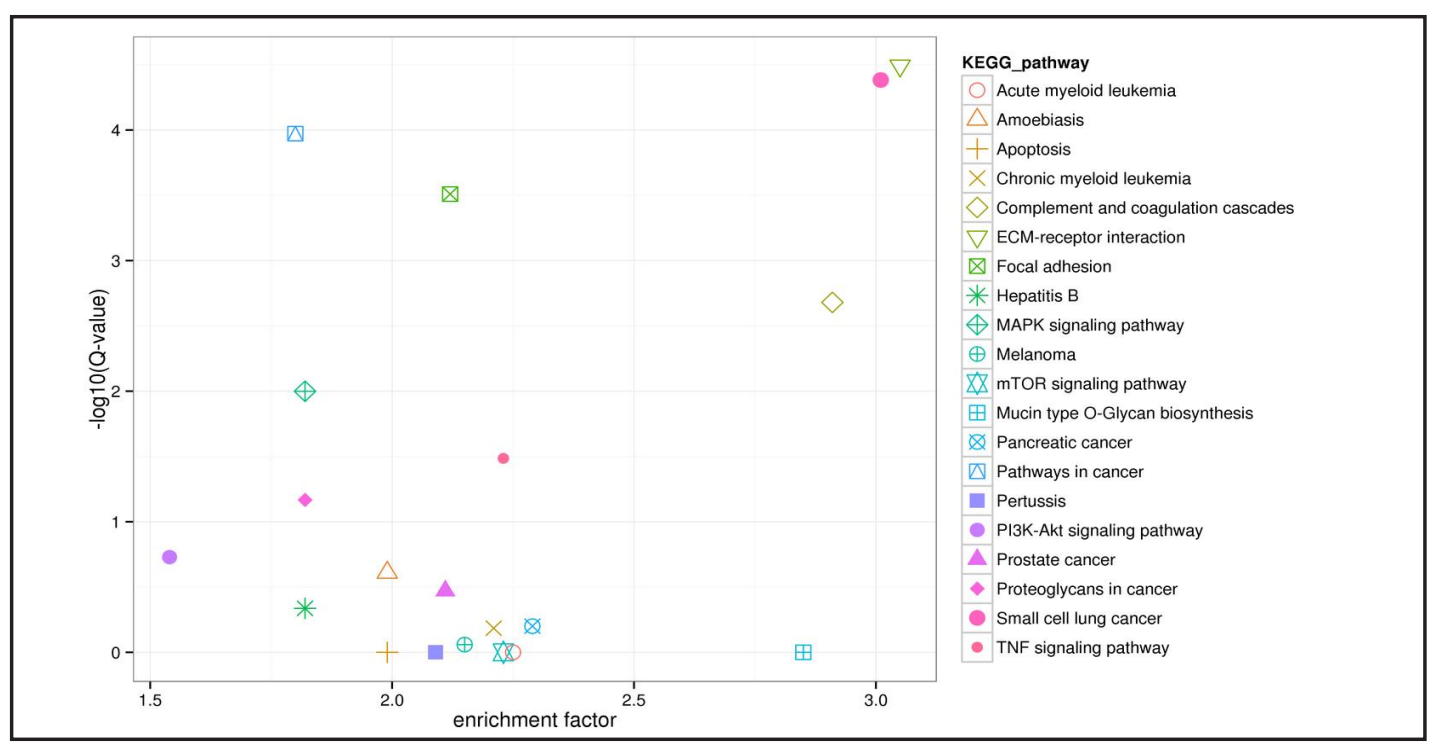

Fig. 3. KEGG pathway enrichment analysis of differentially expressed mRNAs. The first 20 pathways which show the most reliable significance of enrichment(i.e., Q-value) are shown in the graph. Each shape in the graph represents a KEGG pathway whose name is captioned on the right. The $\mathrm{x}$-coordinate is enrichment factor, it represents the ratio of the proportion of differentially expressed genes annotated to the pathway to all genes. The larger the enrichment factor is, the more significant the enrichment level of the differentially expressed genes in the pathway is. The y-coordinate is $-\log _{10}(\mathrm{Q}$-value), the Q-value is the P-value adjusted by multiple hypothesis testing. Thus, the greater the y-coordinate is, the more reliable the enrichment significance of differentially expressed genes in the pathway is. In this figure, the pathways represented by the different shapes in the upper right corner have greater reference value.

ships of miRNA to mRNA and IncRNA to mRNA (Fig. 4), and the protein-protein interactions were also included. To clarify the network diagram, we only included the miRNAs that regulate two or more mRNAs, complete regulatory relationship is shown in Table 5. Nine differently expressed coding RNAs were selected, and GO and KEGG analysis revealed that the functions of these coding RNA involved cell proliferation, metastasis and adhesion, etc. The network indicated that a single coding RNA could be associated with numerous ncRNAs. MiR-200 family, miR-9, miR-128, miR-143/145 cluster, etc. reflect a large range of regulation in the network diagram. Additionally, miR-30e, miR-128 and miR-374 were the miRNAs with the highest expression levels in the two cell lines.

\section{Validation by RT-qPCR}

Validation of the expression patterns of 3 miRNAs, 3 mRNAs and 3 lncRNAs obtained through NGS was performed using RT-qPCR. In all cases, the trend (up-regulation or downregulation) observed in the NGS was consistent with the RT-qPCR results (Table 6).

\section{Discussion}

In cancer research, cell lines are used as tools to explore the mysteries of various diseases, and characterize new therapeutic targets. MCF-7 and MDA-MB-231 cells are the typical cell lines of two clinical breast tumour subtypes. In the present study, we used the NGS method to analyse the coding RNA and ncRNA expression profiles of the two cell lines. The results showed that, compared with MCF-7 cells, there are 540 down-regulated miRNAs in MDAMB-231 cells, including the miR-200 family, miR-145/143 cluster, and miR-7, miR-128, etc., and 41 up-regulated miRNAs including miR-21, miR-10b, miR-210, miR-9, etc. There were 
Fig. 4. Interactions among differentially expressed mRNAs, miRNAs and lncRNAs. (A) and (B) are different ways to show the regulatory relationships, each orange round indicates mRNA, each purple triangle indicates miRNA, and each blue square indicates lncRNA. To make the diagram more clear and concise, we omitted the " $3 p$ " and " $5 p$ " tags of the miRNAs'. The specific names and these RNAs' up- or down-regulation trends are shown in table 5 .

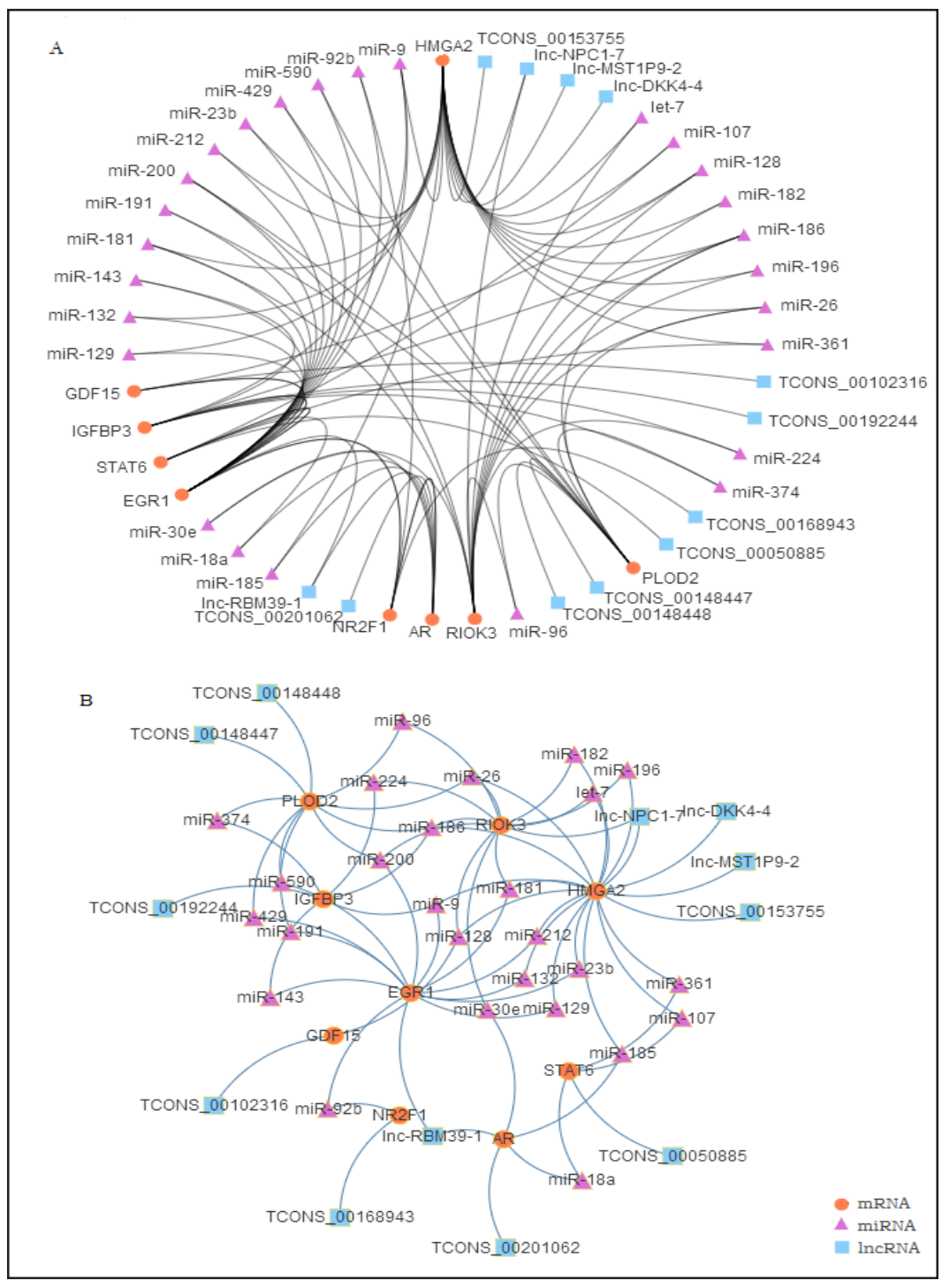

314 up-regulated lncRNAs and 247 down-regulated lncRNAs in MDA-MB-231 cells, including PICSAR and NEAT1. There were 1036 up-regulated mRNAs and 871 down-regulated mRNAs in MCF-7 cells. Among these mRNAs, SLPI, SOD2, etc. were highly expressed in MCF-7, while KRT17, CD55, etc. were highly expressed in MDA-MB-231 cells.

According to the results of the present study, SLPI and SOD2 were highly expressed in MCF-7 cells, and regulated by oestrogen [16, 17]. KRT17 showed a high level of expression in TNBC cells, and the overexpression of BRCA1 significantly reduce the expression of KRT17 [18]. CD55 is a marker of breast cancer stem cells and plays an important role in the tumourigenesis of breast cancer. The high expression of CD55 is an unfavourable prognostic factor [19]. MiR-21 was one of the most highly expressed miRNAs in both cell lines, and its expression was much higher in MDA-MB-231 than in MCF-7 cells. The microRNA target prediction results also showed that miR-21 targeted IGFBP3 (Table 5), which could contribute to the brain metastases of breast cancer cells [20]. MiR-21 levels in tissue and serum have been associated with clinical stage, lymph node metastasis and poor prognosis [21]. MiR-21 can enhance tumour cell proliferation, migration, invasion, survival and the epithelial-to-mesenchymal transition (EMT) through a variety of target genes such as TPM1, PDCD4, and TGF- $\beta 1$. Additionally, knockdown of miR-21 can induce cell apoptosis and inhibit cell proliferation, invasion and EMT. Furthermore, this molecule participates in PTEN, AKT and ERK1/2 signal pathways to promote the generation of breast cancer stem KARGER 


\section{Cellular Physiology Cell Physiol Biochem 2017;44:804-816 \begin{tabular}{l|l|l|} 
DOI: 10.1159/000485344 & O 2017 The Author(s). Published by S. Karger AG, Basel \\
muwwkargercom/cpb
\end{tabular}

Table 5. The complete targeting regulatory relationships of mRNAs and ncRNAs. The "mRNA detail" column lists the mRNAs that have protein-protein interaction with the mRNAs on the left. The "IncRNA detail" column lists the lncRNAs that can target the mRNAs on the left. The "miRNA detail" column lists the miRNAs that can target the mRNAs on the left. " $(\uparrow)$ " represents that RNA is highly expressed in MDA-MB-231 cells compared with MCF7 cells, while " $(\downarrow)$ " means lower expression

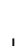

NR2F

$\operatorname{AR}(\downarrow)$;EGR

$1(\uparrow)$

STAT6

$\operatorname{EGR1}(\uparrow)$
CONS 00153755

$\downarrow)$;Inc-NPC1-7( $\downarrow)$ );

nc-MST1P9-2( $\downarrow$ );/n

c-DKK4-4( $\uparrow)$

ER1 $\uparrow$ ) TCONS_00102316

$\uparrow)$

TCONS_00192244(

$\uparrow)$

TCONS_00168943(

$\uparrow$ )

TCONS_00050885(

$\uparrow$ )

TCONS_00148447(

$\downarrow$ );TCONS_0014844

$8(\uparrow)$

Inc-NPC1-7( $\downarrow)$
miRNA_detail

miR-185-5p $(\downarrow)$;miR-18a-5p $(\downarrow)$ );miR-30b-3p $(\downarrow)$ );miR-30c-1-3p $(\downarrow)$;miR-30e$3 p(\downarrow)$;miR-30e-5( $\downarrow)$;miR-425-5p $(\downarrow)$;miR-873( $\uparrow)$

miR-129-5p $(\downarrow)$;miR-132-3p $(\downarrow)$;miR-143-3p $(\downarrow)$;miR-181a-5p $(\downarrow)$ );miR-181b -5p $(\downarrow)$;miR-181c-5p( $\downarrow) ;$ miR-181d-5p $(\downarrow)$;miR-183-5p( $\downarrow)$;miR-191-5p( $\downarrow) ;$ miR-192-5p $(\downarrow)$ );miR-200b-3p $(\downarrow)$;miR-200c-3p( $\downarrow)$;miR-212-3p( $\downarrow)$;miR-215 $-5 p(\downarrow)$;miR-217( $\uparrow$ );miR-23b-3p $(\downarrow)$;miR-32-5p $(\downarrow)$;miR-429( $\downarrow$ );miR-590-3 $\mathrm{p}(\downarrow)$;miR-92b-3p $(\downarrow)$;miR-9-5p( $\uparrow)$

let-7a-2-3p $(\downarrow)$ );let-7a-5p $(\downarrow)$ );let-7b-5p $(\downarrow)$;let-7b-5p $(\downarrow)$ );let-7e-5p $(\downarrow)$;let-7f -5p $(\downarrow)$ );et-7g-5p $(\downarrow)$ );miR-106a $(\downarrow)$ );miR-107( $\downarrow)$ );miR-128-3p $(\downarrow)$ );miR-129-5 $p(\downarrow)$;miR-132-3p $(\downarrow)$ );miR-15a-5p $(\downarrow)$ );miR-15b-5p $(\downarrow)$ );miR-16-5p $(\downarrow)$ );miR-1 7( $\downarrow)$;miR-181a-5p( $\downarrow)$ );miR-181b-5p( $\downarrow) ; m i R-181 c-5 p(\downarrow) ; m i R-181 d-5 p(\downarrow)$; miR-182-5p ( $\downarrow)$ );miR-185-5p $(\downarrow)$;miR-186-5p $(\downarrow)$ );miR-190b( $\downarrow) ;$ miR-195( $\downarrow)$ ;miR-196a-5p ( ) );miR-196b-5p $(\uparrow)$;miR-20a-5p $(\downarrow)$ );miR-212-3p $(\downarrow)$;miR-23 b-3p $(\downarrow) ;$;miR-26a-5p $(\downarrow)$ );miR-26b-5p( $\downarrow)$;miR-361-5p $(\downarrow) ; m i R-362-3 p(\downarrow)$; miR-379-5p $(\downarrow)$;miR-421 $(\downarrow)$;miR-424( $\downarrow)$;miR-503-5p $(\downarrow)$;miR-93-5p $(\downarrow)$;mi $R-9-5 p(\uparrow)$

$\operatorname{miR}-128-3 p(\downarrow)$

miR-125a-5p $(\downarrow)$ );miR-125b-5p $(\downarrow)$ );miR-143-3p $(\downarrow)$ );miR-186-5p $(\downarrow)$ );miR-197 -3p $(\downarrow)$;miR-19a-3p $(\downarrow)$;miR-19b-3p $(\downarrow)$;miR-21-5p $(\downarrow)$;miR-216b-5p $(\downarrow)$;mi R-224-5p $(\downarrow)$;miR-339-5p $(\downarrow)$;miR-340-3p $(\downarrow)$;miR-374a-5p( $\downarrow)$ );miR-374b-5 $\mathrm{p}(\downarrow) ; \mathrm{miR}-9-5 \mathrm{p}(\uparrow)$

miR-92b-5p $(\downarrow)$ miR-107( $\downarrow$ );miR-135b-5p $(\downarrow)$;miR-18a-5p $(\downarrow)$;miR-210-3p $(\uparrow)$ );miR-361-5p( $\downarrow)$;miR-411-5p( $\downarrow)$;miR-486-5p( $\downarrow)$

miR-145-5p $(\downarrow) ;$ miR-186-5p $(\downarrow)$;miR-191-5p $(\downarrow)$;miR-200b( $\downarrow)$;miR-200c( $\downarrow$ );miR-24-3p( $\downarrow$ );miR-26b-5p $(\downarrow)$ );miR-296-3p $(\uparrow)$ );miR-374a-5p( $\downarrow)$ );miR-374b $-3 p(\downarrow) ;$ miR-429( $\downarrow) ;$ miR-590-3p $(\downarrow) ;$ miR-96-5p $(\downarrow)$ let-7a-5p $(\downarrow)$ );let-7b-5p $(\downarrow)$ );let-7b-5p $(\downarrow)$ );let-7e-5p $(\downarrow)$ );let-7f-5p $(\downarrow)$ );let-7g-5 $p(\downarrow)$;miR-128-3p $(\downarrow)$;miR-140-5p $(\downarrow)$;miR-141-3p $(\downarrow)$ );miR-181a-5p( $\downarrow)$;miR -181b-5p( $\downarrow$ );miR-181c-5p $(\downarrow)$ );miR-181d-5p $(\downarrow)$;miR-182-5p $(\downarrow)$;miR-186-5 p( $\downarrow$ );miR-196a-5p $(\downarrow)$ );miR-196b-5p( $\uparrow$ );miR-200a( $\downarrow)$;miR-224-5p( $\downarrow) ; m i R-$ 26a( $\downarrow)$;miR-26b( $\downarrow)$;miR-29a-5p $(\downarrow)$ );miR-29c-3p $(\downarrow)$;miR-30e-5p $(\downarrow)$ );miR-3 20a $(\downarrow) ;$ miR-320b( $\downarrow)$;miR-320c $(\downarrow)$;miR-320d( $\downarrow)$;miR-96-5p( $\downarrow)$

cells [22]. The miR-10b expression in MDA-MB-231 cells was 200 times higher than that in MCF-7 cells. Studies have shown that the up-regulation of miR-10b is closely associated with the invasiveness of breast cancer in breast cancer tissues and cell lines [23]. The miR-10b inhibitor can increase the expression of E-cadherin cells, reduce the expression of vimentin in breast cancer, and decrease invasion and proliferation. The mechanism may be that miR-10b affects the transforming growth factor- $\beta$ (TGF- $\beta$ )-induced EMT [24]. Among over-expressed miRNAs, miR-9 was selected to construct the network. It has been reported that E-cadherin expression mediated by miR-9 decreased the activation of the $\beta$-catenin signalling pathway, which could up-regulate the expression of VEGF to promote tumour angiogenesis [25]. In the network presented herein, miR-9 directly targeted HMGA2, EGR1 and IGFBP3. These 


\section{Cellular Physiology Cell Physiol Biochem 2017;44:804-816

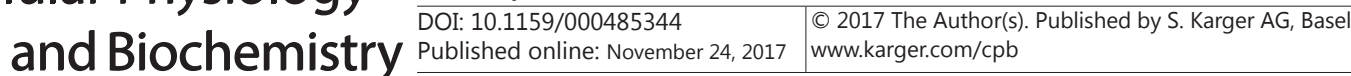

Shi et al.: Differential Expression Profiles of Transcriptome in Breast Cancer Cell Lines

Table 6. Validation of NGS data by real-time qPCR in MCF-7 and MDA-MBA-231 cell lines. ${ }^{*} p<0.001, \log _{2}$ FC: $\log _{2}$ (Fold Change)

\begin{tabular}{|c|c|c|c|c|c|c|c|c|c|c|}
\hline \multirow{2}{*}{\multicolumn{2}{|c|}{$\begin{array}{l}\text { MDA-MB-231 } \\
\text { VS.MCF-7 }\end{array}$}} & \multicolumn{3}{|c|}{ miRNA } & \multicolumn{3}{|c|}{ IncRNA } & \multicolumn{3}{|c|}{ mRNA } \\
\hline & & miR-9-5p & miR-143-3p & miR-145-5p & NEAT1 & PICSAR & GAS5 & CTGF & SPARC & IGFBP2 \\
\hline \multirow{2}{*}{ NGS } & Regulation* & Up & Down & Down & Up & Up & Up & Up & Down & Down \\
\hline & $\log _{2} \mathrm{FC}$ & 3.6 & -6.0 & -5.0 & 6.7 & 6.1 & 3.9 & 9.2 & -9.6 & -7.8 \\
\hline \multirow{2}{*}{ RT-qPCR } & Regulation* & Up & Down & Down & Up & Up & Up & Up & Down & Down \\
\hline & $\log _{2} \mathrm{FC}$ & 3.5 & -8.9 & -8.3 & 4.5 & 2.9 & 2.0 & 7.4 & -7.7 & -.1 \\
\hline
\end{tabular}

three coding RNAs are closely related to the invasion and metastasis of breast cancer [20, 26 , 27]. The expression level of miR-210 in MDA-MB-231 cells is 4 times higher than that in MCF-7 cells in the present study. As its predicted target gene, STAT6 participates in stat6tp63 pathway, which may be involved in inhibiting the metastasis of breast cancer cells to the lung [28]. Previous studies have shown that miR-210, compared with ER+ cells, was highly expressed in TNBC and was described as an independent prognostic factor of TNBC [29].

Among the lower expressed miRNAs, miR-30e, miR-7, miR-200 family and miR-143/145 cluster were selected for further analysis, and these molecules all participated in the network. In MDA-MB-231 cells, miR-30e and miR-7 expression was significantly lower than in MCF-7 cells. A previous study showed that miR-30e may serve as a potential tumour suppressor in breast cancer [30], and this molecule showed lower expression in TNBC compared with non-TNBC patients, and might serve as a new diagnostic marker in TNBC [31]. MiR-7 is negatively correlated with Vimentin mRNA levels in breast carcinoma tissues, inhibited EMT and brain metastatic progression via targeting Focal adhesion kinase (FAK) [32], and could even serve as a biomarker or therapeutic target for brain metastasis [33]. Previous studies have proposed that miR-7 is a tumour suppressor gene, that can be used as a therapeutic target for highly invasive breast cancer [34]. The miR-200 family (miR-200a, miR-200b, and miR-200c) were all highly expressed in MCF-7 compared with the MDA-MB-231 cell line (table 5), and these molecules targeted RIOK3, PLOD2 and EGR1 in the network constructed in the present study. The miR-200 family has been extensively studied in breast cancer research. In different subtypes of breast cancer, as a tumour suppressor [35], the miR-200 family is down regulated in metaplastic carcinoma [36], including the TNBC phenotype. MiR-200c was defined as a luminal cell type-specific microRNA [37]. Decreased expression of the miR-200 family in mammary cancer is associated with lymph node metastasis [38], and their functions are also widely involved in inhibiting the migration [39] and invasion [40] of tumour cells and the resistance of chemotherapeutic agents [41] and radiotherapy [42]. MiR-145/143 is a gene cluster located on chromosome 5q-32-33 and displayed higher expression in luminal-A than in basal-like breast cancer subtypes [43]. Previous studies have shown that the miR-145/143 cluster targets the 3 '-UTR of the ERBB3 gene, thereby simultaneously and cooperatively inhibiting the expression of ERBB3 at the translation level and ultimately inhibiting cancer [44].

Presently, although the research of lncRNA is rapidly developing, there are few studies characterizing the functions of IncRNAs. Most IncRNAs have been demonstrated to control gene expression by regulating various aspects, such as gene methylation, transcriptional activation, and binding to mRNA and miRNA to influence the translation process. Few IncRNAs have been experimentally verified. We only discuss the expression levels of NEAT1 and PICSAR, both of which are highly expressed in MDA-MB-231 cells compared with MCF-7 cells. The hypoxic induction of NEAT1 induced para-speckle formation and the nuclear retention of F11R (also called junctional adhesion molecule 1, JAM1), resulting in the acceleration of tumour proliferation in breast cancer [45]. NEAT1 might also acts as an oncogenic lncRNA and could be regarded as a therapeutic target in human breast cancer [46]. There was a positive correlation between high NEAT1 expression and poor survival in women with breast cancer [45]. The functions of PICSAR (p38 inhibited cutaneous squamous 


\section{Cellular Physiology Cell Physiol Biochem 2017;44:804-816 \begin{tabular}{l|l|l} 
DOI: 10.1159/000485344 & O 2017 The Author(s). Published by S. Karger AG, Basel \\
www.karger.com/cpb
\end{tabular}}

Shi et al.: Differential Expression Profiles of Transcriptome in Breast Cancer Cell Lines

cell carcinoma associated lncRNA) in breast cancer have not been reported. However, studies have shown that PICSAR promotes the growth of cutaneous squamous cell carcinoma by activating the ERK1/2 signal pathway [47]. Similar to PICSAR, some miRNAs were detected in the present study, which have rarely been studied in the field of breast cancer study, but have been shown to play important roles in other fields. For example, miR-361 showed high expression in both MCF-7 and MDA-MB-231 cell lines and has been demonstrated as an antioncogene in lung cancer [48] and endometrial cancer [49], but has not been explored in breast cancer basic research studies. Thus, this molecule may be a novel finding, and further experimental verification is needed.

The up-regulated and down-regulated key genes listed above may explain the stronger invasiveness of the MDA-MB-231 cell line compared with MCF-7 cells. Only some of the results of the present study are inconsistent with those of other scholars, which may be due to the difference between cells and tissues, and the numbers of probes in different versions of the gene chip database are also different. One limitation of the present study is that no normal breast cancer cell lines were included as controls, but we previously elucidated the transcriptome differences between two typical cell lines of breast cancer. Some miRNAs and IncRNAs with significantly differential expression between MCF-7 and MDA-MB-231 cells are less well studied; thus, the present study may provide the basis for further research on breast cancer metastasis and drug resistance.

\section{Acknowledgements}

This work was supported by grants from National Natural Science Foundation of China (81272372 and 30873044 ). The Project-sponsored by SRF for ROCS, SEM.

\section{Disclosure Statement}

The authors declare that there is no conflict of interests regarding the publication of this paper.

\section{References}

1 McGuire A, Brown JA, Malone C, McLaughlin R, Kerin MJ: Effects of age on the detection and management of breast cancer. Cancers (Basel) 2015;7:908-929.

- Mladkova J, Sanda M, Matouskova E, Selicharova I: Phenotyping breast cancer cell lines EM-G3, HCC1937, MCF7 and MDA-MB-231 using 2-D electrophoresis and affinity chromatography for glutathione-binding proteins. BMC Cancer 2010;10:449.

-3 Levenson AS, Jordan VC: MCF-7: the first hormone-responsive breast cancer cell line. Cancer Res 1997;57:3071-3078.

4 Xu J, Huang G, Zhang Z, Zhao J, Zhang M, Wang Y, Liu Z, Lu J: Up-Regulation of Glioma-Associated Oncogene Homolog 1 Expression by Serum Starvation Promotes Cell Survival in ER-Positive Breast Cancer Cells. Cell Physiol Biochem 2015;36:1862-1876.

5 Kwiatkowska E, Wojtala M, Gajewska A, Soszynski M, Bartosz G, Sadowska-Bartosz I: Effect of 3-bromopyruvate acid on the redox equilibrium in non-invasive MCF-7 and invasive MDA-MB-231 breast cancer cells. J Bioenerg Biomembr 2016;48:23-32.

-6 Gest C, Joimel U, Huang L, Pritchard LL, Petit A, Dulong C, Buquet C, Hu CQ, Mirshahi P, Laurent M, FauvelLafeve F, Cazin L, Vannier JP, Lu H, Soria J, Li H, Varin R, Soria C: Rac3 induces a molecular pathway triggering breast cancer cell aggressiveness: differences in MDA-MB-231 and MCF-7 breast cancer cell lines. BMC Cancer 2013;13:63.

7 Liang Z, Wu H, Xia J, Li Y, Zhang Y, Huang K, Wagar N, Yoon Y, Cho HT, Scala S, Shim H: Involvement of miR326 in chemotherapy resistance of breast cancer through modulating expression of multidrug resistanceassociated protein 1. Biochem Pharmacol 2010;79:817-824.

$\checkmark 8$ Chen GQ Zhao ZW, Zhou HY, Liu YJ, Yang HJ: Systematic analysis of microRNA involved in resistance of the 


\section{Cellular Physiology Cell Physiol Biochem 2017;44:804-816 \begin{tabular}{l|l|l} 
and Biochemistry & $\begin{array}{l}\text { DOI: 10.1159/000485344 } \\
\text { Published onlIne: November 24, 2017 }\end{array}$ & $\begin{array}{l}\text { C) } 2017 \text { The Author(s). Published by S. Karger AG, Basel } \\
\text { www.karger.com/cpb }\end{array}$ \\
\hline
\end{tabular}}

MCF-7 human breast cancer cell to doxorubicin. Med Oncol 2010;27:406-415.

-9 Boyerinas B, Park SM, Hau A, Murmann AE, Peter ME: The role of let-7 in cell differentiation and cancer. Endocr Relat Cancer 2010;17:F19-36.

10 Cascione L, Gasparini P, Lovat F, Carasi S, Pulvirenti A, Ferro A, Alder H, He G, Vecchione A, Croce CM, Shapiro CL, Huebner K: Integrated microRNA and mRNA signatures associated with survival in triple negative breast cancer. PLoS One 2013;8:e55910.

11 Huang J, Zhou N, Watabe K, Lu Z, Wu F, Xu M, Mo YY: Long non-coding RNA UCA1 promotes breast tumor growth by suppression of p27 (Kip1). Cell Death Dis 2014;5:e1008.

-12 Augoff K, McCue B, Plow EF, Sossey-Alaoui K: miR-31 and its host gene lncRNA LOC554202 are regulated by promoter hypermethylation in triple-negative breast cancer. Mol Cancer 2012;11:5.

13 Wu Q, Guo L, Jiang F, Li L, Li Z, Chen F: Analysis of the miRNA-mRNA-lncRNA networks in ER+ and ERbreast cancer cell lines. J Cell Mol Med 2015;19:2874-2887.

14 Hurd PJ, Nelson CJ: Advantages of next-generation sequencing versus the microarray in epigenetic research. Brief Funct Genomic Proteomic 2009;8:174-183.

15 Li J, Ma W, Zeng P, Wang J, Geng B, Yang J, Cui Q: LncTar: a tool for predicting the RNA targets of long noncoding RNAs. Brief Bioinform 2015;16:806-812.

16 Liu Z, Gou Y, Zhang H, Zuo H, Zhang H, Liu Z, Yao D: Estradiol improves cardiovascular function through upregulation of SOD2 on vascular wall. Redox Biol 2014;3:88-99.

17 Chen D, Xu X, Cheon YP, Bagchi MK, Bagchi IC: Estrogen induces expression of secretory leukocyte protease inhibitor in rat uterus. Biol Reprod 2004;71:508-514.

18 Gorski JJ, James CR, Quinn JE, Stewart GE, Staunton KC, Buckley NE, McDyer FA, Kennedy RD, Wilson RH, Mullan PB, Harkin DP: BRCA1 transcriptionally regulates genes associated with the basal-like phenotype in breast cancer. Breast Cancer Res Treat 2010;122:721-731.

-19 Ikeda J, Morii E, Liu Y, Qiu Y, Nakamichi N, Jokoji R, Miyoshi Y, Noguchi S, Aozasa K: Prognostic significance of CD55 expression in breast cancer. Clin Cancer Res 2008;14:4780-4786.

20 Saldana SM, Lee HH, Lowery FJ, Khotskaya YB, Xia W, Zhang C, Chang SS, Chou CK, Steeg PS, Yu D, Hung MC: Inhibition of type I insulin-like growth factor receptor signaling attenuates the development of breast cancer brain metastasis. PLoS One 2013;8:e73406.

-21 Yan LX, Huang XF, Shao Q Huang MY, Deng L, Wu QL, Zeng YX, Shao JY: MicroRNA miR-21 overexpression in human breast cancer is associated with advanced clinical stage, lymph node metastasis and patient poor prognosis. RNA 2008;14:2348-2360.

22 Han M, Liu M, Wang Y, Mo Z, Bi X, Liu Z, Fan Y, Chen X, Wu C: Re-expression of miR-21 contributes to migration and invasion by inducing epithelial-mesenchymal transition consistent with cancer stem cell characteristics in MCF-7 cells. Mol Cell Biochem 2012;363:427-436.

-23 Ma L, Teruya-Feldstein J, Weinberg RA: Tumour invasion and metastasis initiated by microRNA-10b in breast cancer. Nature 2007;449:682-688.

24 Han X, Yan S, Weijie Z, Feng W, Liuxing W, Mengquan L, Qingxia F: Critical role of miR-10b in transforming growth factor-beta1-induced epithelial-mesenchymal transition in breast cancer. Cancer Gene Ther 2014;21:60-67.

-25 Ma L, Young J, Prabhala H, Pan E, Mestdagh P, Muth D, Teruya-Feldstein J, Reinhardt F, Onder TT, Valastyan S, Westermann F, Speleman F, Vandesompele J, Weinberg RA: miR-9, a MYC/MYCN-activated microRNA, regulates E-cadherin and cancer metastasis. Nat Cell Biol 2010;12:247-256.

26 Wu J, Zhang S, Shan J, Hu Z, Liu X, Chen L, Ren X, Yao L, Sheng H, Li L, Ann D, Yen Y, Wang J, Wang X: Elevated HMGA2 expression is associated with cancer aggressiveness and predicts poor outcome in breast cancer. Cancer Lett 2016;376:284-292.

27 Wang XX, Cheng Q, Zhang SN, Qian HY, Wu JX, Tian H, Pei DS, Zheng JN: PAK5-Egr1-MMP2 signaling controls the migration and invasion in breast cancer cell. Tumour Biol 2013;34:2721-2729.

28 Papageorgis P, Ozturk S, Lambert AW, Neophytou CM, Tzatsos A, Wong CK, Thiagalingam S, Constantinou AI: Targeting IL13Ralpha2 activates STAT6-TP63 pathway to suppress breast cancer lung metastasis. Breast Cancer Res 2015;17:98.

-29 Radojicic J, Zaravinos A, Vrekoussis T, Kafousi M, Spandidos DA, Stathopoulos EN: MicroRNA expression analysis in triple-negative (ER, PR and Her2/neu) breast cancer. Cell Cycle 2011;10:507-517.

-30 Lin Z, Li JW, Wang Y, Chen T, Ren N, Yang L, Xu W, He H, Jiang Y, Chen X, Liu T, Liu G: Abnormal miRNA-30e Expression is Associated with Breast Cancer Progression. Clin Lab 2016;62:121-128. 


\section{Cellular Physiology Cell Physiol Biochem 2017;44:804-816 \begin{tabular}{l|l|l} 
and Biochemistry & $\begin{array}{l}\text { DOI: 10.1159/000485344 } \\
\text { Published onlIne: November 24, 2017 }\end{array}$ & $\begin{array}{l}\text { C) } 2017 \text { The Author(s). Published by S. Karger AG, Basel } \\
\text { www.karger.com/cpb }\end{array}$ \\
\hline
\end{tabular}}

Shi et al.: Differential Expression Profiles of Transcriptome in Breast Cancer Cell Lines

31 Gasparini P, Cascione L, Fassan M, Lovat F, Guler G, Balci S, Irkkan C, Morrison C, Croce CM, Shapiro CL, Huebner K: microRNA expression profiling identifies a four microRNA signature as a novel diagnostic and prognostic biomarker in triple negative breast cancers. Oncotarget 2014;5:1174-1184.

-32 Kong X, Li G, Yuan Y, He Y, Wu X, Zhang W, Wu Z, Chen T, Wu W, Lobie PE, Zhu T: MicroRNA-7 inhibits epithelial-to-mesenchymal transition and metastasis of breast cancer cells via targeting FAK expression. PLoS One 2012;7:e41523.

-33 Okuda H, Xing F, Pandey PR, Sharma S, Watabe M, Pai SK, Mo YY, Iiizumi-Gairani M, Hirota S, Liu Y, Wu K, Pochampally R, Watabe K: miR-7 suppresses brain metastasis of breast cancer stem-like cells by modulating KLF4. Cancer Res 2013;73:1434-1444.

-34 Zhang H, Cai K, Wang J, Wang X, Cheng K, Shi F, Jiang L, Zhang Y, Dou J: MiR-7, inhibited indirectly by lincRNA HOTAIR, directly inhibits SETDB1 and reverses the EMT of breast cancer stem cells by downregulating the STAT3 pathway. Stem Cells 2014;32:2858-2868.

35 Blick T, Hugo H, Widodo E, Waltham M, Pinto C, Mani SA, Weinberg RA, Neve RM, Lenburg ME, Thompson EW: Epithelial mesenchymal transition traits in human breast cancer cell lines parallel the CD44(hi/)CD24 (lo/-) stem cell phenotype in human breast cancer. J Mammary Gland Biol Neoplasia 2010;15:235-252.

-36 Castilla MA, Diaz-Martin J, Sarrio D, Romero-Perez L, Lopez-Garcia MA, Vieites B, Biscuola M, RamiroFuentes S, Isacke CM, Palacios J: MicroRNA-200 family modulation in distinct breast cancer phenotypes. PLoS One 2012;7:e47709.

-37 Bockmeyer CL, Christgen M, Muller M, Fischer S, Ahrens P, Langer F, Kreipe H, Lehmann U: MicroRNA profiles of healthy basal and luminal mammary epithelial cells are distinct and reflected in different breast cancer subtypes. Breast Cancer Res Treat 2011;130:735-745.

-38 Xu F, He H, Huang W, Lin Y, Luo S, Du Q, Duan R: Decreased expression of MicroRNA-200 family in human breast cancer is associated with lymph node metastasis. Clin Transl Oncol 2016;18:283-288.

-39 Korpal M, Lee ES, Hu G, Kang Y: The miR-200 family inhibits epithelial-mesenchymal transition and cancer cell migration by direct targeting of E-cadherin transcriptional repressors ZEB1 and ZEB2. J Biol Chem 2008;283:14910-14914.

40 Burk U, Schubert J, Wellner U, Schmalhofer O, Vincan E, Spaderna S, Brabletz T: A reciprocal repression between ZEB1 and members of the miR-200 family promotes EMT and invasion in cancer cells. EMBO Rep 2008;9:582-589.

41 Chen J, Tian W, Cai H, He H, Deng Y: Down-regulation of microRNA-200c is associated with drug resistance in human breast cancer. Med Oncol 2012;29:2527-2534.

42 Lin J, Liu C, Gao F, Mitchel RE, Zhao L, Yang Y, Lei J, Cai J: miR-200c enhances radiosensitivity of human breast cancer cells. J Cell Biochem 2013;114:606-615.

43 Li D, Xia H, Li ZY, Hua L, Li L: Identification of Novel Breast Cancer Subtype-Specific Biomarkers by Integrating Genomics Analysis of DNA Copy Number Aberrations and miRNA-mRNA Dual Expression Profiling. Biomed Res Int 2015;2015:746970.

44 Yan X, Chen X, Liang H, Deng T, Chen W, Zhang S, Liu M, Gao X, Liu Y, Zhao C, Wang X, Wang N, Li J, Liu R, Zen K, Zhang CY, Liu B, Ba Y: miR-143 and miR-145 synergistically regulate ERBB3 to suppress cell proliferation and invasion in breast cancer. Mol Cancer 2014;13:220.

-45 Choudhry H, Albukhari A, Morotti M, Haider S, Moralli D, Smythies J, Schodel J, Green CM, Camps C, Buffa F, Ratcliffe P, Ragoussis J, Harris AL, Mole DR: Tumor hypoxia induces nuclear paraspeckle formation through HIF-2alpha dependent transcriptional activation of NEAT1 leading to cancer cell survival. Oncogene 2015;34:4482-4490.

46 Lo PK, Zhang Y, Wolfson B, Gernapudi R, Yao Y, Duru N, Zhou Q: Dysregulation of the BRCA1/long noncoding RNA NEAT1 signaling axis contributes to breast tumorigenesis. Oncotarget 2016;7:65067-65089.

-47 Piipponen M, Nissinen L, Farshchian M, Riihila P, Kivisaari A, Kallajoki M, Peltonen J, Peltonen S, Kahari VM: Long Noncoding RNA PICSAR Promotes Growth of Cutaneous Squamous Cell Carcinoma by Regulating ERK1/2 Activity. J Invest Dermatol 2016;136:1701-1710.

-48 Hou XW, Sun X, Yu Y, Zhao HM, Yang ZJ, Wang X, Cao XC: miR-361-5p suppresses lung cancer cell lines progression by targeting FOXM1. Neoplasma 2017;64:526-534.

49 Ihira K, Dong P, Xiong Y, Watari H, Konno Y, Hanley SJ, Noguchi M, Hirata N, Suizu F, Yamada T, Kudo M, Sakuragi N: EZH2 inhibition suppresses endometrial cancer progression via miR-361/Twist axis. Oncotarget 2017;8:13509-13520. 